\title{
A RULE-BASED APPROACH TO MODULAR SYSTEM DESIGN
}

\author{
Francesco Parisi-Presicce \\ Dipartimento di Matematica Pura ed Applicata \\ Universita' degli Studi - L'Aquila \\ I-67100 L'Aquila (Italy)
}

\begin{abstract}
The problem of designing a modular software system which realizes a given specification starting from a predefined set of abstract data types is reduced to the problem of deriving the goal specification using the productions and then translating the derivation sequence (if it exists) into an interconnection of algebraic module specifications. The approach is based on the existence of a library of reusable modules, each represented by its visible interfaces in the form of productions.
\end{abstract}

\section{INTRODUCTION}

The development of correct programs is very difficult (if at all possible) without an appropriate formal support. Such a support can be provided by the algebraic approach with simple semantics and the central role played in algebraic specifications by the concept of abstract data type and the support this concept gives to the decomposition of large systems ([5]) . For basic data types, a simple algebraic specification with sorts and operations with their equational properties is sufficient; the choice of a unique (up to isomorphism) algebra allows to abstract with respect to the data representation. In order to abstract from part of a specification to make it "variable" (parameter), it is necessary to use parametrized specifications along with the appropriate mechanisms for parameter passing [10]. For large software systems, it is useful to have another form of abstraction in order to hide internal details and to specify only the properties necessary for the interface between the user and the implementor. A formal concept of modules with an export interface was first proposed in [15]. The addition of an import interface and a parameter part leads to our notion of module specification ([10], [22], [1]). In our framework, a module specification consists of four parts : an import interface specifying what the module "needs" to operate, an export interface specifying the data type "produced" and made available to the outside by the module, a parameter part shared by the interfaces, and a body part in which the sorts and operations of the export interface are implemented in terms of those of the import interface. The items in the body and not in the export are hidden. The behaviour (or semantics) of a module is a transformation from data types which satisfy the import specification to data types which satisfy the export specification.

In order to develop large software systems in a stepwise manner, a flexible system of interconnections is needed to form the horizontal structuring of the system. The main interconnections viewed as operations on modules are : union, where the corresponding parts of two modules are put together by specifying the common subpart to be identified; composition, where the export interface of a module is matched with the import interface of another module; actualization, where the parameter part is replaced by an actual specification. These (and other [17], [18]) mechanisms guarantee the "correctness" of the result given the correctness of the arguments and the compositionality of the semantics ([1], [11]).

There is an analogy between this notion of module and that of MODULA-2 and ADA. A MODULA-2 module is subdivided into a "definition module", the visible part, and an "implementation module", corresponding roughly to our export interface and body part. The interconnection mechanism in MODULA-2 is "strict" : the union is realized implicitly (syntactically) while for the composition, modules can import other items, not by specifying the properties that they must satisfy but by referencing explicitly other modules. More details in [14] . A similar comparison with ADA has been reported in [1], where packages correspond to modules with an empty parameter and generics to the more general case. In both cases, only syntactic aspects, i.e., types and operations, of a component specification are allowed, leaving it to comments in a natural language to convey their meaning to user and implementor (with the obvious danger of incompleteness and misinterpretation). In our algebraic approach, semantical aspects are included in the specification of the interfaces. In the simplest framework of the basic algebraic case, the four parts are represented by algebraic specifications, with equations or positive conditional equations as axioms, and are related by specificaton morphisms. The formalism has been extended to include algebraic constraints and first order logical formulas ([8]) or specifications in more general institutions.

The import and export interfaces, along with the shared parameter, are the only part visible to the outside and provide the gates through which each module interacts with the other ones. The basic idea proposed is to use the interfaces as productions or rules and to apply them to a specification. Given a library of (correct) modules represented by their interfaces as productions of specifications, the problem of developing the horizontal structure for a modular system to realize a GOAL specification given a set of predefined data types is viewed as the problem of symbolically deriving (a specification containing) the GOAL specification from the specification PRE of the predefined types using the given productions. To each derivation and each combination of derivations corresponds an operation on the module specifications which realize the productions used. The sequence of derivations from PRE to GOAL can be translated into a modular design whose behavior is to transform any model of PRE into a model (realization) of the objective GOAL. The discussion here is restricted to the derivations and the translations from the derivations to module design. We postpone to future papers other important aspects such as 
strategies of search or choice of applicable productions based on problem specific knowledge.

R.M.Burstall and J.A.Goguen :

Another important factor for the practical utilization of abstract specification languages is to build up a library of specifications which can then be used in putting together other large specifications. .... Without such a library, every program specification effort will have to start from scratch and there will be no significant progress...

\section{MODULES AND PRODUCTIONS}

In this section, we briefly review some basic notions of algebraic specifications [10] and the concept of module specification ([1]) with the operations used for the horizontal structuring mechanism of module specifications. For ease of presentation, we consider only the basic algebraic case of module specifications without constraints (in the sense of [8]). By algebraic specification SPEC we mean a triple $(S, O P, E)$ consisting of a set $S$ of sorts, a set OP of operation symbols and a set $\mathrm{E}$ of (positive conditional) equations. To distinguish among the parts of different specifications, we subscript the components, i.e. refer for example to the second component of the algebraic specification SPEC1 as OPSPEC1 . Sig(SPEC) denotes the signature (S,OP) of SPEC and, for $N \in O P$, sorts $(\mathrm{N})$ denotes the set of sorts of the operator symbol $\mathrm{N}$. $\mathrm{Eqn}(\mathrm{Sig}(\mathrm{SPEC})$ ) is the set of equations over (S, OP) and a set $X$ of variables. A specification morphism $\mathrm{f}: \mathrm{SPECl} \rightarrow$ SPEC2 is a signature morphism (fS,fOP) : (S1,OP1) $\rightarrow$ (S2,OP2) which associates in consistent way operator symbols to operator symbols and sorts to sorts and such that the translation $\mathrm{f}^{\#}(\mathrm{E} 1)$ of the equations of SPEC1 is contained in E2 (it can be extended [19] to $f^{\#}(E 1)$ derivable from E2 or to equationally closed sets $E$ of equations). Each specification morphism $\mathrm{f}: \mathrm{SPEC} 1 \rightarrow$ SPEC2 defines a forgetful functor $\mathrm{V}_{\mathrm{f}}$ $\mathrm{Alg}$ (SPEC2) $\rightarrow \mathrm{Alg}$ (SPEC1) (which intuitively translates models of SPEC2 into models of SPEC1 disregarding possible additions introduced by $f$ ) and a free functor $F_{f}$ : $\mathrm{Alg}$ (SPEC1) $\rightarrow \mathrm{Alg}$ (SPEC2) (which intuitively builds for each model A of SPEC1 the model of SPEC2 with the least amount of properties compatible with the specification SPEC2, using the elements of $A$ as constants). In all our examples, we use the following more intuitive notation, listing under the key words sorts, opns and eqns the elements of the three components of the specification.

FS-PAR $=$ bool +

sorts $\mathrm{f}^{*}$, dest, dep

opns EQF : f* f* $\rightarrow$ bool

NODEP : $\rightarrow$ dep

eqns $\mathrm{EQF}\left(\mathrm{F}^{*}, \mathrm{~F}^{*}\right)=$ TRUE

denotes the specification obtained by adding to the standard specification of boolean values the set of sorts $S^{\prime}=\left[\mathrm{f}^{*}\right.$, dest, dep \} , the two operation symbols EQF and NODEP (the second one with an empty argument list of sorts denotes a constant $)$ and the equation $\mathrm{EQF}\left(\mathrm{F}^{*}, \mathrm{~F}^{*}\right)=$ TRUE universally quantified over all the $F^{*}$ ranging over elements of sort $\mathrm{f}^{*}$. Considering the obvious inclusion of bool in FS-PAR as a specification morphism $\mathrm{f}$, the forgetful functor $\mathrm{V}_{\mathrm{f}}$ applied to any model of FS-PAR returns its boolean part ignoring all elements of sorts $\mathrm{f}^{*}$, dest or dep.
2.1 Definition [13], [1]

A module specification MOD consists of four algebraic specifications PAR (parameter part), EXP (export interface), IMP (import interface) and BOD (body) and four specification morphisms as in the following commutative diagram

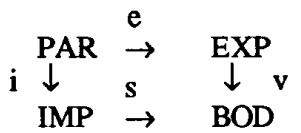

A module specification is correct if the free functor $F_{S}: A l g(I M P) \rightarrow A l g(B O D)$ satisfies $V_{S}\left(F_{S}(A)\right)=A$ for all IMP-algebras A. The semantics SEM of MOD is the functor $\mathrm{V}_{\mathrm{V}} \mathrm{F}_{\mathrm{S}}: \operatorname{Alg}(\mathrm{IMP}) \rightarrow \operatorname{Alg}(\mathrm{EXP})$.

Interpretation We say that MOD realizes its interface Int(MOD) $=(\mathrm{IMP} \leftarrow \mathrm{PAR} \rightarrow \mathrm{EXP})$ which represents the only information available outside the module. Both the export and the import interface are "contained" in the body specification, which provides an implementation of EXP by IMP. The operations and sorts in BOD but not in EXP are to be considered hidden. The semantics of MOD is a transformation between algebras : the import specifies the kind of algebra to be provided to the module to obtain an algebra which satisfies the export interface. The export and import interfaces share a parameter part, which, if the module is correct, is the only part of any IMP-algebra A guaranteed to be left unchanged by the semantical transformation. If $\mathrm{PAR}=\mathrm{IMP}$ and $\mathrm{EXP}=\mathrm{BOD}$, this notion coincides with that of parametrized specification.

\subsection{Example}

The example of a module for a flight schedule consisting of triples with flight number ( $\left.f^{*}\right)$, destination and departure, is taken from [1]. It avoids duplication of flight numbers with the export equation on ADD-FS, which is implemented in the body by the hidden operation $\mathrm{TAB}$ which keeps an unordered list of triples. FS-MOD =

(FS-PAR, FS-EXP, FS-IMP, FS-BOD)

where FS-PAR is the specification given above, FS-IMP is exactly FS-PAR, while

FS-EXP = FS-PAR +

sorts fs

opns CREATE-FS : $\rightarrow$ fs

SEAR-FS : $f^{*}$ fs $\rightarrow$ bool

ADD-FS : $f^{*}$ dest dep fs $\rightarrow$ fs

RET-FS : f* fs $\rightarrow$ dep

CHAN-FS : $f^{*}$ dep fs $\rightarrow$ fs

eqns

SEAR-FS $\left(F^{*}\right.$, CREATE-FS $)=$ FALSE

SEAR-FS $\left(\mathrm{F}^{*}, \mathrm{FS}\right)=\mathrm{TRUE}=>$

SEAR-FS $\left(F^{*}, F S\right)=$ TRUE $\Rightarrow$

ADD-FS $\left(F^{*}\right.$, DEST, DEP, FS $)=$ FS

RET-FS(F*,CHANGEFS(F*,DEP,FS $))=$ DEP

and

FS-BOD $=$ FS-EXP +

opns TAB : $f^{*}$ dest dep fs $\rightarrow$ fs

eqns ADD-FS $\left(F^{*}\right.$, DEST,DEP,CREATE-FS $)=$ TAB $\left(F^{*}, D E S T, D E P, C R E A T E-F S\right)$

$\mathrm{ADD}-\mathrm{FS}\left(\mathrm{F}^{*} 1, \mathrm{DE} 1, \mathrm{DEP} 1, \mathrm{TAB}\left(\mathrm{F}^{*}, \mathrm{DE}, \mathrm{DEP}, \mathrm{FS}\right)\right)=$

if $\mathrm{EQF}\left(\mathrm{F}^{*} 1, \mathrm{~F}^{*}\right)$ then $\mathrm{TAB}\left(\mathrm{F}^{*}, \mathrm{DE}, \mathrm{DEP}, \mathrm{FS}\right)$

else TAB $\left(F^{*}, \mathrm{DE}, \mathrm{DEP}, \mathrm{ADD}-\mathrm{FS}\left(\mathrm{F}^{*} 1, \mathrm{DE} 1, \mathrm{DEP} 1, \mathrm{FS}\right)\right)$

SEAR-FS $\left(\mathrm{F}^{*}, \mathrm{CREATE}-\mathrm{FS}\right)=\mathrm{FALSE}$

SEAR-FS(F*,TAB(F*1,DEST,DEP,FS $))=$ $\mathrm{EQF}\left(\mathrm{F}^{*}, \mathrm{~F}^{*} 1\right)$ or SEAR-FS $\left(\mathrm{F}^{*}, \mathrm{FS}\right)$ 


\author{
RET-FS(F*,CREATE-FS $)=$ NODEP \\ RET-FS $\left(\mathrm{F}^{*}, \mathrm{TAB}\left(\mathrm{F}^{*} 1, \mathrm{DEST}, \mathrm{DEP}, \mathrm{FS}\right)\right)=$ if $\mathrm{EQF}\left(\mathrm{F}^{*}, \mathrm{~F}^{*} 1\right)$ \\ then DEP else RET-FS(F*,FS) \\ CHAN-FS(F*,DEP,CREATE-FS) $=$ CREATE-FS \\ CHAN-FS(F*,DEP,TAB(F*1,DEST1,DEP1,FS) $=$ \\ if $\mathrm{EQF}\left(\mathrm{F}^{*}, \mathrm{~F}^{*} 1\right)$ then $\mathrm{TAB}\left(\mathrm{F}^{*}, \mathrm{DEST} 1, \mathrm{DEP}, \mathrm{FS}\right)$ else \\ $\mathrm{TAB}\left(\mathrm{F}^{*} 1, \mathrm{DEST} 1, \mathrm{DEP} 1, \mathrm{CHANFS}\left(\mathrm{F}^{*}, \mathrm{DEP}, \mathrm{FS}\right)\right)$
}

The four specification morphisms as in 2.1 are all inclusions. The operation TAB in the body is used to implement the exported operation ADD but is not visible to the outside. The semantics of the module reflects the fact that the operations in the body not already in the import have only the properties specified by their (equational) definitions and their consequences (in the usual equational calculus) of these definitions. In other words, the defining equations are to be seen as rewrite rules to compute the value of the functions. With this view of the body equations, the correctness of the module specification corresponds to requiring that the rewrite rules just mentioned define a total function (termination and confluency of the rules) if the result is of a sort of the import algebra. No such restrictions are imposed on operations with result value of a new sort.

There are three basic operations which, when applied to module specifications and parametrized specifications, yield new module specifications. Such operations guarantee the correctness of the resulting module if the argments are correct and allow to express the semantics of the result in terms of the semantics of the arguments of the operation. We describe these operations informally, referring the reader to [1], [11] and [14] for details and the formal definition.

The operation of union MOD1 +MOD0 MOD2 of two module specifications MOD1 and MOD2 with respect to a common submodule MODO is obtained by taking for each component of the result the disjoint union of the corresponding components of MOD1 and MOD2 and then identifying their common part of MOD0. Only MOD0 (possibly up to renaming) determines the parts not to be duplicated. The resulting module specification is automatically correct and its semantics is given by the "amalgamated sum" SEM1+SEM0 SEM2 of the corresponding semạtics ([2], [11]).

The operation of actualization acth(PS, MOD) consists of replacing the parameter part PAR of MOD by a (parametrized) specification $P S=(P a r, A C T)$ via a parameter passing morphism h:PAR $\rightarrow$ ACT which associates the actual sorts and operations of ACT with the formal ones of PAR. The replacement of PAR by ACT is propagated to the interfaces and the body part (since PAR is "contained" in all three). Any new import algebra is composed of an ACT-algebra and an IMP-algebra : the semantics of the actualized module acth(PS, MOD) leaves unchanged the ACT-part and transforms the IMP-part according to SEM ([16],[11]).

$$
\sqrt{\sqrt{ }} \begin{gathered}
\text { PAR - EXP } \\
1 \\
\text { IMP - BOD }
\end{gathered}
$$

The third basic operation on module specifications is that of composition MOD1·MOD2, where the import interface of a module specification is "matched" with the export interface of another one via a specification morphism h. The "unused" interfaces provide two of the components of the composite module specification and the "intersection" of the parameters is the new parameter. The new body is the body BOD1 where IMP1 has been replaced by BOD2. The sorts and the operations of EXP2 are no longer exported by the composite module, whose semantics is the composition of the original ones, interposed by the possible translation of the matched interfaces ( SEM1 $\left.\cdot \mathrm{V}_{\mathrm{hE}} \cdot \mathrm{SEM} 2\right)$. The resulting module is again correct if MOD1 and MOD2 are ([10],[1])

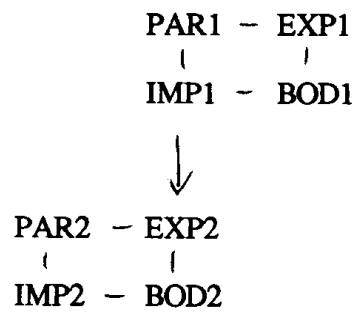

These basic operations, along with the other ones of product, partial composition, recursion (cyclic dependencies of modules) and iteration satisfy several algebraic laws which allow different but semantically equivalent strategies for interconnecting module specifications.

Each module consists of four parts, but only three of them, namely the two interfaces and the parameter part, are visible to the system designer or in general to the user of the module specification. The body contains the construction of the export interface operations in terms of those of the import interface and the internal representation of the exported data . Only the designer of the module has access to the body of the module. The interfaces (and their shared parameter part) provide the "gates" through which each module interacts with the other modules and contain the information about the module behavior which is visible to the user of the module. So there is a clear separation between what the module does (i.e. transforms IMP-algebras into EXP-algebras) and the details of how this transformation is implemented (in the body). The interfaces of a module specification then provide the information necessary to reason about its properties and behavior and that are relevant fto the reuse of the module. Our approach to system design is based on viewing the interfaces of a module specification as a production and on applying these productions to specifications. If starting from an initial specification, the goal specification is generated using the productions, then the goal specification can be realized through a modular system built using the realizations of the productions (library modules) and the way in which the productions are applied (interconnection of modules).

\subsection{Definition [19]}

A SPEC-production is an ordered pair PRO = ( IMP $\leftarrow$ PAR $\rightarrow$ EXP ) of injective specification morphisms i : PAR $\rightarrow$ IMP and e:PAR $\rightarrow$ EXP . A direct derivation via the production $P R O$ consists of the following two pushout diagrams of specifications 


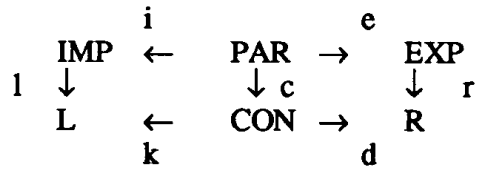

A production PRO is applicable to a specification $L$ if there exist a morphism $1:$ IMP $\rightarrow \mathrm{L}$ and a context specification CON such that $\mathrm{L}$ is the pushout (gluing) of IMP and CON. The result $R$ of the derivation is the pushout of EXP and CON. In this case we write $P R O: L \Rightarrow R$ and say that $R$ is derivable from L via PRO.

Interpretation The middle specification PAR represents the part of IMP left unchanged by the production. The specification CON in the direct derivation is the part of $L$ not affected by the derivation : it is the "context" of the derivation and it is "glued" to the right end side EXP of the production via the common subspecification PAR, which represents the "interface" between the unchanged context and the modified part (from IMP to EXP). Notice that a direct derivation is symmetric and that if $\mathrm{PRO}: \mathrm{L} \Rightarrow \mathrm{R}$, then $\mathrm{PRO}^{-1}: \mathrm{R} \Rightarrow \mathrm{L}$, where $\mathrm{PRO}^{-1}=(\mathrm{EXP} \leftarrow \mathrm{PAR} \rightarrow \mathrm{IMP})$.

\subsection{Example}

The interfaces of the FS-MOD of Example 2.2 along with the obvious inclusions form a production of algebraic specifications FS-PRO. When this production is applied to the specification $L=$ nat + string where

$$
\begin{aligned}
& \text { nat }=\text { bool }+ \\
& \text { sorts nat } \\
& \text { opns } \quad 0: \rightarrow \text { nat } \\
& \text { succ : nat } \rightarrow \text { nat } \\
& \mathrm{Eq}: \text { nat nat } \rightarrow \text { bool } \\
& \text { eqns } E q(\operatorname{succ}(x), \operatorname{succ}(y))=E q(x, y) \\
& \mathrm{Eq}(\mathrm{x}, \mathrm{x})=\text { TRUE } \\
& \operatorname{Eq}(0, \operatorname{succ}(x))=\text { FALSE } \\
& \operatorname{Eq}(\operatorname{succ}(x), 0)=\text { FALSE } \\
& \text { string = sorts } \quad \text { alph, str } \\
& \text { opns EMPTY : } \rightarrow \text { str } \\
& \text { MAKE : alph } \rightarrow \text { str } \\
& \text { eqns } \\
& \text { CONC : str str } \rightarrow \text { str } \\
& \operatorname{CONC}(\operatorname{CONC}(x, y), z)=\operatorname{CONC}(x, \operatorname{CONC}(y, z)) \\
& \operatorname{CONC}(E M P T Y, x)=x=\operatorname{CONC}(x, \text { EMPTY })
\end{aligned}
$$

via the specification morphism 1: FS-IMP $\rightarrow$ nat+string with $1 S\left(f^{*}\right)=$ nat $=1 S($ dep $), 1 S($ dest $)=s t r$, loP(EQF $)=E q$, lOP(NODEP $)=E M P T Y$, it produces the specification

$\mathrm{R}=\mathrm{L}+$ sorts $\mathrm{fs}$

opns CREATE-FS : $\rightarrow$ fs

SEAR-FS : nat $f s \rightarrow$ bool

ADD-FS : nat str nat $f s \rightarrow$ fs

RET-FS : nat $f s \rightarrow$ dep

CHAN-FS : nat nat $f s \rightarrow f s$

eqnS

SEAR-FS $(\mathrm{N}$, CREATE-FS $)=$ FALSE

SEAR-FS $(N, F S)=T R U E \Rightarrow$ ADD-FS $(N$, ST1, ST2, FS $)=F S$
SEAR-FS $(N, F S)=$ TRUE $\Rightarrow$ RET-FS(N, CHAN-FS(N,ST1,FS))=ST1

Notice that in this example the effect of applying the production is to add to the specifications of nat and string the five operator symbols labelled with -FS . This is the case when the production is deductive, i.e., when PAR=IMP. In this case, it is sufficient to have a specification morphism

$1:$ IMP $\rightarrow$ L to obtain a direct derivation via the production. In general, a production PRO $=($ IMP $\leftarrow$ PAR $\rightarrow$ EXP $)$ is applicable to the specification $L$ if there exists a context specification CON and a specification morphism

c : PAR $\rightarrow$ CON such that $L=I M P+P A R C O N$, i.e., if $L$ can be decomposed into two parts IMP and CON which share exactly the PAR part of the production. PAR represents the "boundary" between the part IMP involved in the derivation via PRO and the part CON not affected by the production. The result of the derivation is then the specification obtained by "gluing" CON and EXP via PAR. In general, the "pushout complement" CON = L - PAR IMP need not exist. Intuitively, the specification CON should contain all the sorts of PAR and the sorts of $L$ not contained in IMP, i.e., SCON $=S_{L}-1 S\left(S_{I M P}-\right.$ is $\left.\left(S_{P A R}\right)\right)$. Similarly for the operator symbols $O_{C O N}=O P P L_{1}-$ lOP $_{\text {OPIMP }}$ - iOP(OPPAR $)$ ). It is obvious that for $\mathrm{CON}$ to be well defined, the operator symbols cannot use sorts not contained in the set of sorts $\mathrm{S}_{\mathrm{CON}}$. Similarly, the equations $\mathrm{E}_{\mathrm{CON}}=\mathrm{E}_{\mathrm{L}}$ -

$1^{\#}\left(E_{\text {IMP }}-i^{\#}\left(E_{P A R}\right)\right)$ of CON cannot be formed using operator symbols not in OPCON if we want a properly defined specification. If the "occurrence" morphism

$1:$ IMP $\rightarrow \mathrm{L}$ is injective, this is all that is needed for 1 to be applicable. If not, then there are some technical conditions that require the items collapsed by 1 to be "gluing" items.

\subsection{Theorem}

If $1:$ IMP $\rightarrow \mathrm{L}$ is a specification morphism for the production $\mathrm{PRO}=(\mathrm{IMP} \leftarrow \mathrm{PAR} \rightarrow \mathrm{EXP})$ and

$\mathrm{DANG}=\{\mathrm{s} \in$ SIMP: $\mathrm{N} \in$ OPL- $\mathrm{lOP}(\mathrm{OP}$ IMP) and $1 \mathrm{~s}(\mathrm{~s}) \in \operatorname{sorts}(\mathrm{N})\}$ IDS $=\left\{s \in\right.$ SIMP $\left._{\text {IM }} \mathrm{s}^{\prime} \in \mathrm{SIMP}, \mathrm{s}^{\prime} \neq \mathrm{s}, \mathrm{lS}_{\mathrm{S}}(\mathrm{s})=1 \mathrm{~s}\left(\mathrm{~s}^{\prime}\right)\right\}$

IDOP $=\left\{N \in\right.$ OPIMP : N' $\in$ OPIMP, N $\neq \mathbf{N}^{\prime}, l_{O P}(\mathbf{N})=1$ OP $\left.\left(N^{\prime}\right)\right\}$. then the existence of CON such that $L=I M P+P A R C O N$ is equivalent to the following three conditions

1) is $($ SPAR $) \supset D A N G \cup I D S$

2) iOP(OPPAR) $\supset$ IDOP

3) Eqn ((SCON, OPCON)) $\mathrm{E}_{\mathrm{CON}}$

\section{FROM DERIVATION TO DESIGN}

Suppose now that we have been able to generate the goal specification from the initial one using as productions the interfaces of the library modules. How can we now use the way the goal is derived to design a modular system which realizes this goal, that is, which transforms a model of the initial specification into a model of the goal specification? The modular system is built using the definition of applicability of a production and the way parallel and concurrent derivations appear in the generation.

First notice that if $R$ is derivable from $L$ via the production $\mathrm{PRO}=(\mathrm{IMP} \leftarrow \mathrm{PAR} \rightarrow \mathrm{EXP})$ then any L-algebra is the amalgamated sum ([1]) of an IMP-algebra I and a CON-algebra $\mathrm{C}$ via the common PAR-algebra $\mathrm{P}$, written $\mathrm{I}+\mathrm{P} \mathrm{C}$. 
CON specifies the part of $L$ left unchanged by the derivation, either because in PAR or because outside the part affected by IMP. Therefore the derivability of $R$ from $L$ induces a semantical transformation from $I+P C$ to $\operatorname{SEM}(I)+P C$, the only one compatible with the semantical transformation of PRO form I to SEM(I). All this is just the general case of the (specific) interpretation of derivation as operation on module specification. Recall that MOD realizes PRO if $\mathrm{PRO}=\operatorname{Int}(\mathrm{MOD})$.

\subsection{Theorem [19]}

If $R$ is derivable from $L$ via the production $P R O=$ (IMP $\leftarrow$ PAR $\rightarrow$ EXP) as in 2.3 and MOD realizes PRO, then there exists a module specification MOD' trasforming Lalgebras into R-algebras. The module MOD' consists of the module MOD where the parameter PAR has been actualized by CON via c : PAR $\rightarrow$ CON. Its semantics SEM' is given by $\operatorname{SEM}^{\prime}(\mathrm{I}+\mathrm{P} C)=\operatorname{SEM}(\mathrm{I})+\mathrm{P} C$.

Going back to Example 2.4, the derivability of $\mathrm{R}$ from $\mathrm{L}$ via the interfaces of FS-MOD implies the existence of a module act 1 (nat + string,FS-MOD) which has nat + string as import and as export interface the specification

$$
\mathrm{R}=\mathrm{L}+\text { sorts fs }
$$

opns CREATE-FS : $\rightarrow$ fs

SEAR-FS : nat $\mathrm{fs} \rightarrow$ bool

ADD-FS : nat str nat $\mathrm{fs} \rightarrow \mathrm{fs}$

RET-FS : nat fs $\rightarrow$ dep

CHAN-FS : nat nat fs $\rightarrow$ fs eans SEAR-FS $(N$, CREATE-FS $)=$ FALSE

SEAR-FS(N,FS) $\Rightarrow$ ADD-FS(N, ST1, ST2, FS) $=$ FS SEAR-FS(N,FS) $=$ TRUE $\Rightarrow$

$$
\text { RET-FS(N, CHAN-FS(N,ST1,FS)) }=S T 1
$$

In order to translate a derivation sequence into system design, we must investigate the relationship between the interaction of two derivations and that of the two modules realizing the corresponding productions.

The simplest case is that of a derivation sequence PRO1 : $L \Rightarrow R, P R O 2: R \Rightarrow R^{\prime}$ where the overlap of the two productions in $\mathbf{R}$ consists of common gluing elements only, i.e., when r1(e1(PAR1)) $\cap$ 12(i2(PAR2)) つ r1(EXP1) I2(IMP2) . Such sequence is called sequentially independent In this case, the production PRO2 does not need the elements added to $\mathrm{R}$ by the first production PRO1 and can therefore be applied directly to $\mathrm{L}$ to produce $\mathrm{R}^{\prime \prime}$. The two direct derivations PRO1: $L \Rightarrow R$ and PRO2 : $L \Rightarrow R$ " so obtained are parallel independent , i.e., they too intersect only in common gluing elements and can therefore be applied in parallel (simultaneously). This discussion is summarized in the next theorem which needs the definition of subproduction. A production PRO0 is a subproduction of PRO1 if there exist specification morphisms as in the following commutative diagrams

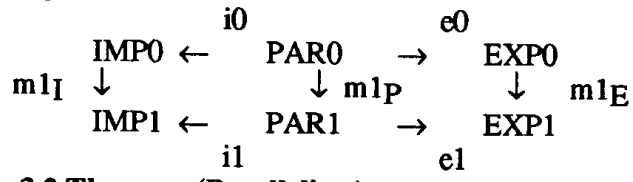

\subsection{Theorem (Parallelism)}

Let $\mathrm{PRO} 1: \mathrm{L} \Rightarrow \mathrm{R}$ and $\mathrm{PRO} 2: \mathrm{R} \Rightarrow \mathrm{R}^{\prime}$ be a sequentially independent derivation sequence. Then
1) PRO2 can be applied to $L$ producing a specification $R^{\prime \prime}$

2) the derivations PRO1: $L \Rightarrow R$ and $P R O 2: L \Rightarrow R^{\prime \prime}$ are parallel independent

3) for some subproduction $\mathrm{PRO0}$ of $\mathrm{PRO} 1$ and $\mathrm{PRO} 2$ with IMP0 $=P A R 0=E X P 0$, the parallel production $\mathrm{PRO1}+\mathrm{PRO0} \mathrm{PRO} 2$ is applicable to $\mathrm{L}$ and PRO1 +PRO0 PRO2 : L $\Rightarrow$ R'.

Consider as production the following interfaces PS-PRO of a module specification which builds a plane schedule $(\mathrm{ps})$ with no duplications of the plane number $\left(\mathrm{p}^{*}\right)$.

PS-IMP = PS-PAR = bool +

sorts $\mathrm{p}^{*}$, type, seats

opns EQP : $\mathrm{p}^{*} \mathrm{p}^{*} \rightarrow$ bool

eans $\mathrm{EQP}\left(\mathrm{P}^{*}, \mathrm{P}^{*}\right)=\mathrm{TRUE}$

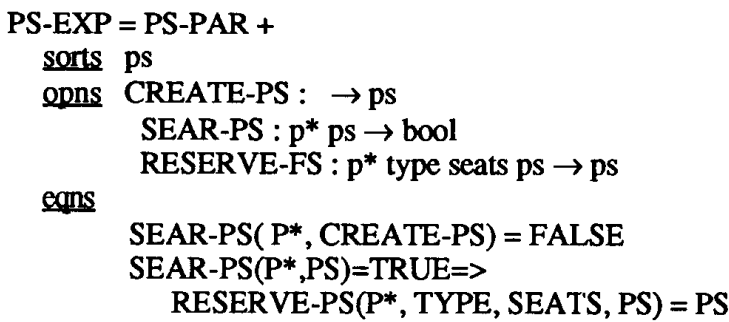

As shown in Example 2.4, the production FS-PRO can be applied to $L=$ nat + string producing the specification $R$ shown there. The production PS-PRO can now be applied to $R$ via the specification morphism $12:$ PS-IMP $\rightarrow R$ with which $12 \mathrm{~S}\left(\mathrm{p}^{*}\right)=12 \mathrm{~S}$ (seats) = nat, $12 \mathrm{~S}$ (type) $=$ str and $12 \mathrm{OP}(\mathrm{EQP})=\mathrm{Eq}$

Notice that since PS-PRO is deductive, there is no need to check the conditions of Theorem 2.5 .

The application of PS-PRO to $R$ produces the specification

$\mathbf{R}^{\prime}=$ nat + bool +

sorts fs, ps

opns CREATE-FS : $\rightarrow \mathrm{fs}$

SEAR-FS : nat fs $\rightarrow$ bool

ADD-FS : nat str nat fs $\rightarrow$ fs

RET-FS : nat $\mathrm{fs} \rightarrow$ nat

CHAN-FS : nat nat $f s \rightarrow f s$

CREATE-PS : $\rightarrow$ ps

SEAR-PS : nat ps $\rightarrow$ bool

RESERVE-FS : nat str nat ps $\rightarrow$ ps

eqns SEAR-FS( N, CREATE-FS) = FALSE

SEAR-FS(N,FS)=TRUE $\Rightarrow$

SEAR-FS(N,FS) $=$ TRUE $=$ ADD-FS(N, ST1, ST2, FS) $=$ FS RET-FS(N, CHAN-FS(N,ST,FS))=ST

SEAR-PS( N, CREATE-PS) $=$ FALSE

SEAR-PS(N1,PS) $=$ TRUE $\Rightarrow$ RESERVE-PS(N1,STR,N2, PS) =PS

The two derivations FS-PRO : $\mathrm{L} \Rightarrow \mathrm{R}$ and

PS-PRO : $R \Rightarrow R$ ' are sequentially independent because the images of FS-EXP and PS-IMP in R overlap only in nat + string which in turn is contained in the image of the gluing part (FS-PAR and PS-PAR) of each of the two productions. By Theorem 3.2 we can anticipate the application of PS-PRO directly to $L$ using the same specification morphism 12 defined above. The subproduction PRO0 of (3) of the theorem above 
is just $\mathrm{PROO}=($ bool $\leftarrow$ bool $\rightarrow$ bool $)$ and the parallel production FSPS-PRO = FS-PRO +PRO0 PS-PRO is given by

FSPS-PAR = FSPS-IMP = FS-IMP+bool PS-IMP

FSPS-EXP = FS-EXP +bool PS-EXP

By applying the parallel production FSPS-PRO to $\mathrm{L}$ we obtain in one direct derivation the specification $R^{\prime}$ above.

Given that the original productions are the interfaces of module specifications from the library, we can use these modules to build (define) another one which realizes the parallel production FSPS-PRO. If we call by Mbool the module specification where each part is the boolean specification bool and each morphism the identity, then the parallel production FSPS-PRO consists of the interface of the union FS-MOD +Mbool PS-MOD of the module specifications FS-MOD and PS-MOD with respect to their shared (subparameter) part Mbool ([16],[11]). Combining this with Theorem 3.1, we obtain a module specification act 1 (nat + string, FS-MOD + Mbool PS-MOD) whose import and export specifications are $L$ and $R^{\prime}$ respectively. Note that we could have obtained the same result by first taking the disjoint union of the modules FS-MOD and PSMOD and then actualizing the result by the actual specification nat + string : this actualization identifies the two copies of bool coming from the two distinct modules. Similarly, we could have taken the disjoint union FS-PRO + PS-PRO of the productions in the Parallelism Theorem : the subproduction of (3) of that theorem is not unique and can be chosen in a certain range (between the empty specification and exactly the overlap of the two derivations). The corresponding modules are different, but the subsequent actualized ones are the same.

The parallel production of Theorem 3.2(3) is a special case of a more general operation on productions : amalgamation. If we start with a subproduction PRO0 of PRO1 and PRO2 we can define the amalgamation of PRO1 and PRO2 w.r.t. PRO0 as the production (IMP1+IMP0 IMP2 $\leftarrow$ PAR1 +PAR0 $\mathrm{PAR} 2 \rightarrow \mathrm{EXP1+EXP0} \mathrm{EXP2)} \mathrm{denoted} \mathrm{by} \mathrm{PRO1+PRO0}$ $P R O 2$. The UNION Theorem of [19] states that if $P R O 0$ is a sub-production of PRO1 and PRO2 and each production $P R O j$ is applicable to a specification $L j$ to produce the specification $\mathrm{Rj}$ in a "consistent" way (that is the effect of PRO1 and PRO2 on the common subpart LO is the same and exactly that of PRO0) then PRO1+PRO0 PRO2 : L1+LO L2 $\Rightarrow R 1+R 0 R 2$ i.e., consistent productions on parts ( $L 1$ and L2) of the same specification ( $\mathrm{L} 1+\mathrm{L} 0 \mathrm{L2}$ ) can be amalgamated into one production.

Another interaction between sequential derivations that can be translated into an interconnection of module specifications is matching. A pair of derivations PRO1 : $\mathrm{L} 1 \Rightarrow \mathrm{L} 2$ and PRO2 : L2 $\Rightarrow \mathrm{L} 3$ is matched if EXP1=IMP2 and the morphisms r1 : EXP1 $\rightarrow$ L2 and $12:$ IMP2 $\rightarrow$ L2 are the same. For such a sequence of matched derivations, we can construct a new production which can accomplish the same global transformation. The composition of two productions PRO1 $=($ IMP1 $\leftarrow$ PAR $1 \rightarrow$ EXP1 $)$ and PRO2 $=(I M P 2 \leftarrow$ PAR2 $\rightarrow$ EXP2) is defined when EXP1=IMP2, it is denoted by PRO2-PRO1 and is given by (IMP1 $\leftarrow$ PAR3 $\rightarrow$ EXP2) where PAR3 is the pullback (intersection) of PAR1 and PAR2 in EXP1. As might be expected, the result of applying the composite production $\mathrm{PRO} 2 \cdot \mathrm{PRO} 1$ is the same as the sequential application of the two productions (first PRO1 and then PRO2)

\subsection{Theorem(Matched Concurrency)}

Let PRO1 : L1 $\Rightarrow \mathrm{L} 2$ and PRO2 : L2 $\Rightarrow \mathrm{L} 3$ be a pair of matched derivations. Then the composition of PRO1 and $\mathrm{PRO} 2$ can be applied to $\mathrm{L} 1$ and $\mathrm{PRO} 2 \cdot \mathrm{PRO} 1: \mathrm{L} 1 \Rightarrow \mathrm{L} 3$.

To illustrate the result of the Theorem, suppose that we have another library module whose interface consists of APS-PAR = FS-PAR + bool PS-PAR

APS-IMP $=$ FS-EXP + bool PS-EXP

APS-EXP $=$ APS-PAR +

Sorts aps

opns CREATE : $\rightarrow$ aps

SEAR : $f^{*}$ aps $\rightarrow$ bool

RET : $f^{*}$ aps $\rightarrow$ dep

CHAN : $f^{*}$ dep aps $\rightarrow$ aps

SCHED : $f^{*}$ dest dep $p^{*}$ type seats aps $\rightarrow$ aps

eqns

$\operatorname{SEAR}\left(\mathrm{F}^{*}, \mathrm{CREATE}\right)=\mathrm{FALSE}$

$\operatorname{SEAR}\left(\mathrm{F}^{*}, \mathrm{APS}\right)=\mathrm{TRUE}=>$ SCHED $\left(F^{*}, \mathrm{D}, \mathrm{DEP}, \mathrm{P}^{*}, \mathrm{TYPE}, \mathrm{SEATS}, \mathrm{APS}\right)=\mathrm{APS}$

$\operatorname{SEAR}\left(\mathrm{F}^{*}, \mathrm{APS}\right)=\mathrm{TRUE}=>$ $\operatorname{RET}\left(\mathrm{F}^{*}, \mathrm{CHAN}\left(\mathrm{F}^{*}, \mathrm{DEP}, \mathrm{APS}\right)\right)=\mathrm{DEP}$

This production can be applied to the specification $R^{\prime}$ by the morphism $12:$ APS-IMP $\rightarrow$ R' given by

$12 S\left(f^{*}\right)=12 S\left(p^{*}\right)=12 S($ dep $)=12 S($ seats $)=$ nat;

$12 \mathrm{~S}(\mathrm{dest})=12 \mathrm{~S}(\mathrm{type})=\mathrm{str} ; 12 \mathrm{~S}(\mathrm{fs})=\mathrm{fs} ;$ etc.

The result of such a direct derivation is the specification

L3 = nat + string +

sorts aps

opns CREATE : $\rightarrow$ aps

SEAR : nat aps $\rightarrow$ bool

RET : nat aps $\rightarrow$ nat

CHAN : nat nat aps $\rightarrow$ aps

SCHED : nat str nat nat str nat aps $\rightarrow$ aps

eqns

SEAR(N,CREATE) $=$ FALSE

SEAR(N,APS) $=$ TRUE $=>$

SCHED(N1,ST1,N2,N3,ST2,N4,APS)=APS

SEAR(N,APS) $=$ TRUE $\Rightarrow$ $\operatorname{RET}\left(\mathrm{N}, \mathrm{CHAN}\left(\mathrm{N}, \mathrm{N}^{\prime}, \mathrm{APS}\right)\right)=\mathbf{N}^{\prime}$

The direct derivations FSPS-PRO : nat + string $\Rightarrow R^{\prime}$ and APS-PRO : $\mathbf{R}^{\prime} \Rightarrow$ L3 satisfy the hypothesis of the Concurrency Theorem. We can therefore define the composition APS-PRO FSPS-PRO of the productions to obtain the production AFPS-PRO =

(AFPS-IMP $\leftarrow$ AFPS-PAR $\rightarrow$ AFPS-EXP) where

AFPS-IMP $=$ FSPS-IMP

AFPS-PAR $=$ APS-PAR and

AFPS-EXP $=$ APS-EXP.

We can now apply the production AFPS-PRO to the initial specification nat + string to obtain in only one derivation the specification L3 above. The module specification corresponding to this derivation is the actualization by nat + string of the composition of the APS-MOD module which realizes the APS production above, with the union module FS-MOD +Mbool PS-MOD. 
The Matched Concurrency Theorem deals only with the simplest case of interaction between two sequential derivations (besides of course the sequentially independent case). Consider two derivations PRO1 : L1 $\Rightarrow \mathrm{L} 2$ and PRO2 : L2 $\Rightarrow \mathrm{L} 3$ and a "relation" for them, i.e., a specification REL and two specification morphisms b1 : REL $\rightarrow$ EXP1 and

b2 $:$ REL $\rightarrow$ IMP2 such that in the diagram

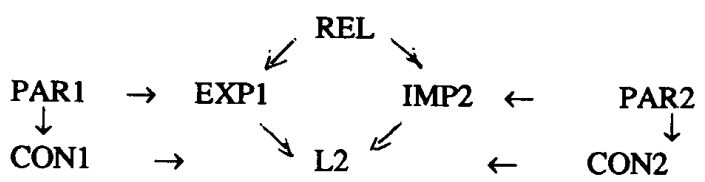

the middle diamond commutes. The matched Concurrency Theorem deals with the case where REL $=$ EXP1 $=$ IMP2 and the two morphisms are the identity. There are more general cases where the two derivations can be combined into one using a composite production.

Two similar and symmetric cases are those where either $\mathrm{REL}=\mathrm{EXP} 1$ or $\mathrm{REL}=\mathrm{IMP} 2$. In the first case, EXP1 is only part of the specification IMP2 and therefore the part of IMP2 not provided for by PRO1 must be "pushed back" and joined with IMP1. The IMP-part of the composite production consists then of IMP1 and IMP2 - b2(EXP1), its gluing part of the common part of PAR1 and PAR2 and its EXP-part consists of EXP2 only (since everything "generated" by PRO1 has been "used" by PRO2 via the morphism b2). The new production can be realized by taking the partial composition ([17]) of the realizations of the productions PRO1 and PRO2. The symmetric case where REL=IMP2 can be constructed in a similar way, "adding" for the final EXP-part the specification EXP2 and the part EXP1 - b1(IMP2) of EXP1 not "used" by $\mathrm{PRO} 2$. The realization of this composite production can be accomplished by using the operation of partial product ([18]) on the single realizations.

The most general case of an arbitrary relation REL for a sequence of derivations has been considered in [12] and has required a composite interconnection mechanism to construct its realization from the realizations of the component productions.

\section{ADAPTING THE DESIGN}

It is quite unlikely that the library at our disposal contains all the productions needed to generate our goal specification. More likely is the situation where part of the goal specification can be realized using the given modules and part must be implemented anew. We illustrate the situation with an example. Call SPEC the following specification of a system to handle the activity of a library such as build and manipulate a list of books and a list of patrons, associate borrowed books with borrowers, keep track of the due dates for the RET of borrowed books, etc.

SPEC $=$ bool +

sorts title, author, duedate, books,

patno ( number identifying a patron),

type (of patron), phone,patrons, library

opns CREATE: $\rightarrow$ books

LOOK : title books $\rightarrow$ bool

RESERVED : title author duedate books $\rightarrow$ books

DATE: title books $\rightarrow$ duedate
UPDATE : title duedate books $\rightarrow$ books

NODATE : $\rightarrow$ duedate

EQT : title title $\rightarrow$ bool

NEW : $\rightarrow$ patrons

FIND : patno patrons $\rightarrow$ bool

ADD : patno type phone patrons $\rightarrow$ patrons

START: $\rightarrow$ library

RETURNED : title library $\rightarrow$ library

SEAR : title library $\rightarrow$ bool

BORROW: title author duedate patno type phone library $\rightarrow$ library

eqns SEAR(TI,START) $=$ FALSE

$\operatorname{SEAR}(T I, \mathrm{LIB})=\mathrm{TRUE}=>$

BORROW(TI,AUT,DD,PN,TYP,PHO,LIB)=LIB

LOOK $(T I, B K)=$ TRUE $\Rightarrow$ DATE(TI,UPDATE $(T I, D D, B K))=$ DD

LOOK $(T I, B K)=$ TRUE $\Rightarrow>$ RESERVED $(T I, A U T, D D, B K)=B K$

LOOK $($ TI, CREATE $)=$ FALSE

EQT(TI,TI) $=$ TRUE

FIND(PN,NEW) $=$ FALSE

FIND(PN,PAT) $=$ TRUE $=>$ ADD $(P N, T Y P, P H O, P A T)=P A T$

RETURNED(TI,START) $=$ START

RETURNED(TI,BORROW(TIL,AUT,DD,PN,TYPPHO,LIB))

$=$ if EQT(TI,TIL) then LIB else

BORROW(TL,AUT,DD,PN,TYP,PHO,RETURNED(TI,LIB)

We have listed the sorts by their intuitive name to simplify the reading. The elements of sort title, author and type are intended to be strings, while duedate, patno and phone are to be represented as natural numbers. The specification GOAL to be realized is SPEC with such a substitution.

Now we want to know if and how this specification can be realized using the library of modules represented by the productions FS-PRO, PS-PRO and APS-PRO illustrated in the previous sections and starting from the initial specification nat + string .

As we have seen in section 2, we can apply FS-PRO to nat + string to produce

SP1 = nat + string +

sorts fs

opns CREATE-FS : $\rightarrow$ fs

SEAR-FS : nat $f s \rightarrow$ bool

ADD-FS : nat str nat fs $\rightarrow$ fs

RET-FS : nat $\mathrm{fs} \rightarrow$ dep

CHAN-FS : nat nat $f s \rightarrow f s$

eqnS

SEAR-FS ( N, CREATE-FS) $=$ FALSE

SEAR-FS(N,FS) $=$ TRUE $\Rightarrow>$ ADD-FS $(N, S T 1, S T 2, F S)=F S$

SEAR-FS(N,FS) $=$ TRUE $=>$

RET-FS(N, CHAN-FS(N,ST1,FS))=ST1

To this specification we can apply, as illustrated in section 3, the production PS-PRO to obtain

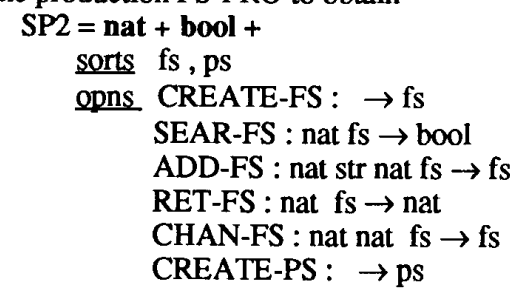


SEAR-PS : nat ps $\rightarrow$ bool

RESERVE-FS : nat str nat $\mathrm{ps} \rightarrow \mathrm{ps}$

equs

SEAR-FS(N,FS) $=$

SEAR-FS(N,FS) $=$ TRUE $=>$ ADD-FS(N, ST1, ST2, FS $)=$ FS

RET-FS(N, CHAN-FS(N,ST,FS)) $=S T$

SEAR-PS $(\mathrm{N}, \mathrm{CREATE}-\mathrm{PS})=$ FALSE

SEAR-PS(N1,PS) $=>$

RESERVE-PS(N1,STR,N2,PS)= PS

Now we can apply the production APS-PRO to generate the specification

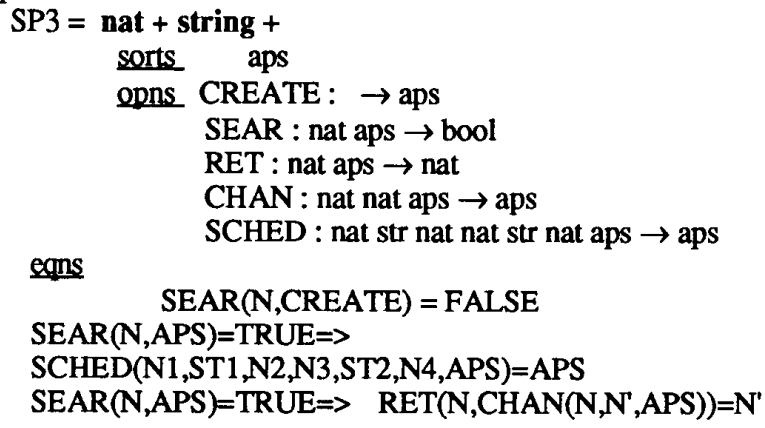

Finally we can apply again FS-PRO and PS-PRO (in any order since they are sequentially independent) to SP3 to obtain the specification

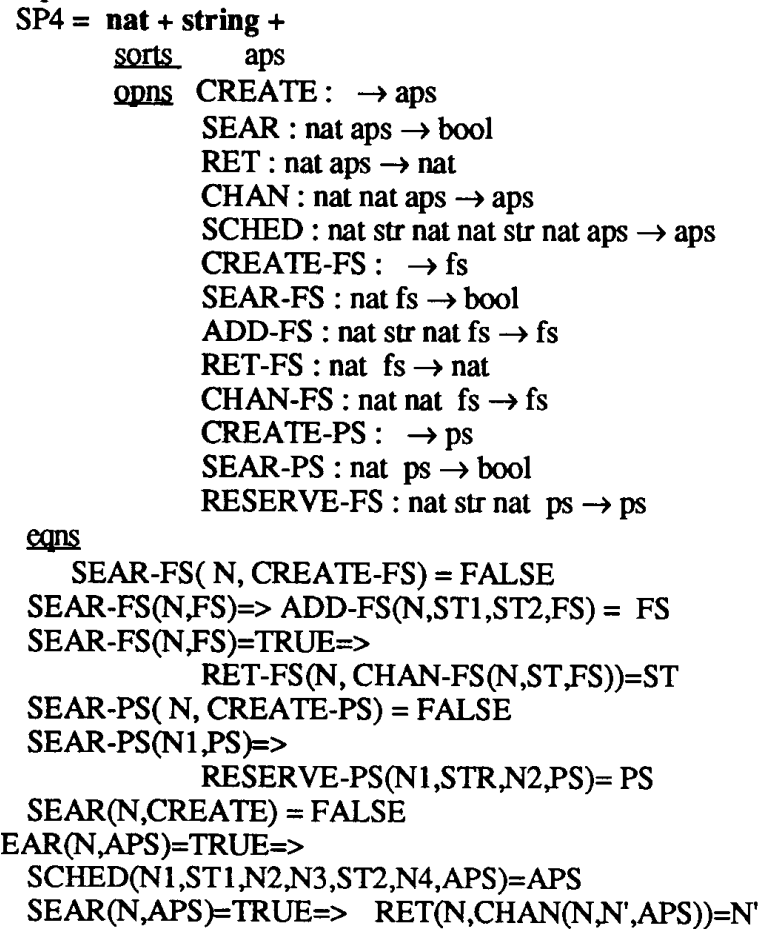

Notice now that with a simple renaming (fs stands for books, ps for patrons and aps for library) which can be accomplished by a production $\mathrm{REN}=$ ( OLDNAMES $\leftarrow$ OLDNAMES $\rightarrow$ NEWNAMES) where the first morphism is the identity and the second one the one-to-one and onto mapping performing the renaming, we have our GOAL specification with the exception of the operator RETURNED. We have generated the specification SP4 while what is required is the specification

$\mathrm{GOAL}=\mathrm{SP} 4+$

opns RETURNED : str $\rightarrow$ aps

eans RETURNED(TI,START)=START

RETURNED(TI,BORROW(TIL,AUT,DD,PN,TYPPHO,LIB))

= if EQT(TI,TIL) then LIB else

BORROW(TIL,AUT,DD,PN,TYP,PHO,RETURNED(TI,LIB)

Using the Parallelism Theorem and the Matched Concurrency Theorem of the last section, we can define the modular system which realizes the specification SP4 by taking :

- FIRST the union FS-MOD + Mbool PS-MOD = FSPSMOD of the modules (realizing the productions FS-PRO and PS-PRO used in the first 2 steps) which when actualized by nat + string would give SP2 as export interface;

- THEN the composition AFPS-MOD = APS-MOD -FSPSMOD of the module obtained in the previous step and the module realizing the production APS-PRO (the specification SP3 can be obtained as export after actualizing this composite module by nat + string)

- THEN, with the Parallelism Theorem again, the union FSMOD + Mbool PS-MOD of the realizations of the productions FS-PRO and PS-PRO used to generate SP4 from SP3 and combine this module with the one used to generate SP3 by taking their union (one more application of the Parallelism Theorem)

We can now exploit the compatibility of the actualization of module specifications with other operations on modules $([16],[11])$ and the connection, expressed in Theorem 3.1, between a derivation via a production and the actualization of the realization of the production. By actualizing the last module specification obtained by the parameter passing morphism 1: PAR $\rightarrow$ nat + string, we obtain the module act 1 ( nat + string,

(APS-MOD• FSPS-MOD) +Mbool FSPS-MOD)

whose export interface contains the specification SP4.

We are in a much better position than we were at the beginning : instead of constructing a module specification to implement, starting from nat+string, fourteen operations satisfying the properties expressed in the specification SPEC, we only need to implement the operation called RETURNED, the only one not contained in our generated specification. The problem of obtaining the operation RETURNED can be solved in (at least) three different ways, all of which entail the addition, via extension, of the operation to the modular system.

\section{- DIRECT EXTENSION}

This approach is the most immediate one and it consists of adding the operation to the export interface and (hence) to the body part. At this point, an attempt is made at defining the operation RETURNED in terms of the other operations present in the body (which contains all the operations from import and export) and then to show that the properties which RETURNED must satisfy are a consequence of the equations satisfied by the other operators. Since new operations and 
equations are added to the body of the module, the correctness of the new module must be proven. Unfortunately, the extension is not a "clean" operation (in the sense of [7]) in that it does not guarantee the correctness of the result based on the correctness of the parts. We must then prove the correctness property of 2.1 and the task is not trivial because it involves the body of the total module. To the difficulty of proving correctness, we must add the possibility that the new operation cannot be implemented in terms of the import operations. This situation would then require an extension of the import interface and, along with the proof of correctness as before, a revision of the applicability of the overall module to the specification of the predefined types. One more drawback of this approach is the loss of modularity of the system : the resulting module cannot in general be decomposed into the union or composition of self-contained modules and the new operation is reusable only to the extent that the whole module is . In our example there is no need to add anything to the import of the module. The GOAL specification can be realized as the export interface of act 1 ( nat+string, ext (AFPS-MOD +Mbool FSPS-MOD, (RETURNED: ... ))

\section{- PARTIAL EXTENSION}

This approach consists of extending only part of the module specification constructed so far. The basic idea is that RETURNED is part of a subspecification SP of GOAL. Since only the operation RETURNED of GOAL has not been generated, there must be a point in the derivation where the subspecification SP - \{RETURNED\} is derived. It is at this point in the generation that we can extend the module by adding to it the missing operation. We search therefore for the "closest" derivation to SP and add RETURNED to the modular system designed up to that point. In our example, RETURNED is part of the subspecification containing START, BORROW and SEAR and therefore can already be added to the realization of SP3. The GOAL specification can be realized as the export interface of act 1 (nat+string, ext (AFPS-MOD, (RETURNED ... ) + Mbool FSPS-MOD )) As in the previous case, there is no need to add new items to the import interface to implement RETURNED but the (necessary) correctness proof is simpler than in the DIRECT EXTENSION because the module to be proven correct is smaller (AFPS-MOD instead of AFPS-MOD+Mbool FSPSMOD ). Furthermore, the system has maintained some of its modular structure, in particular all the modular structure deriving from subsequent applications of productions. Since the modules realizing these last productions are not affected by the extension, no new proof of their correctness ie required.

\section{-CLEAN EXTENSION}

This is the most desirable way of adding the part of the GOAL specification not generated. It consists of designing a new module which implements RETURNED and the smallest part of SP necessary to have a well defined specification. This new module is then combined (using the union operation) with the module realizing the rest of SP (and with which it shares a common submodule). The new module, smaller in general than the one obtained with PARTIAL EXTENSION, must be proven correct and compatible, in its shared part, with the one already realizing the rest of SP. Besides the smaller correctness proof, this approach preserves the modular structure of the system since the missing items are added to the rest using clean operations such as union and composition. In our particular example, this approach does not give the expected improvements over the PARTIAL EXTENSION approach. This is due to the fact that the equations characterizing RETURNED contain occurrences of the operations START, EQT and BORROW and the axiom of the last one contains SEAR as well: there is no meaningful decomposition of the specification that will lead to a nontrivial union of two modules. What can be done is to notice that, since the addition of RETURNED does not require an extension of the import of APS-MOD, we can restrict our extension to this last module and then compose it with the realization of the productions used earlier. Using this observation, we obtain GOAL as the export interface specification of the module

act 1 (nat+string, (ext (APS-MOD , (RETURNED ...)) FSPS-MOD) +Mbool FSPS-MOD ))

\section{CONCLUDING REMARKS}

In this paper, we have proposed an approach to the development of software systems based on ideas and techniques from the algebraic theory of Graph Grammars ([6]). Given an initial specification, SPEC-productions are applied sequentially or in parallel to generate another specification : if the productions are the interfaces of module specifications from a library, then the derivation sequence can be translated into a modular system. The results obtained are intended as a formal support for a rule-based (expert) system to aid the systematic development of large software systems from a library of reusable components. We have discussed in details the notions of derivability and of translation of a derivation sequence into an interconnection of modules and we have seen how to anticipate the application of a production using the Parallelism Theorem. This equivalence of derivations translates into provably equivalent modular systems. There may be several derivation sequences which generate the GOAL specification and each may give a different system, with different characteristics such as size of modules, number of interconnections, etc. Even for a given sequence, there may be different systems realizing the GOAL : we are investigating ([12]) the manipulation of a given sequence into "canonical" form to obtain a canonical system with the smallest number of interconnections.

We have discussed how to apply a rule and how to translate the result, but not the strategies necessary in the choice of the rule. The development could be purely syntactic, trying all possible rules, or could be directed, possibly in an interactive way, by knowledge specific to the problem, giving priority to the rules based on semantical aspects not represented in the specification. Along the same lines, we have assumed the existence of an "occurrence" morphism $1:$ IMP $\rightarrow$ L and then established the conditions for the applicability of the production. The choice of 1 could also be directed by other semantical conditions. Furthermore, the possibility may arise that a given morphism is consistent with respect to sorts and operations but not with respect to the axioms : at this point a design decision is needed on whether or not to add the property to what is being realized.

We have discussed the horizontal structure of the system and how to recover it from the derivation. What needs to be investigated also is how the derivation (and hence the system) must change to accomodate the "vertical" development ([7]) of 
modules and system, with notions such as refinement and simulation. We have pointed out the symmetry of the direct derivation in 2.3. This symmetry allows us to perform a search of the GOAL specification in a generative or forward mode similar to the derivation of a string in a grammar or in a goal-oriented or backward mode starting from the GOAL and applying the productions from EXP trying to arrive at base cases of predefined types (more on this in a forthcoming paper) - Also the derivation sequence can be used as documentation on the decomposition of the final system.

A module specification can be viewed as the description of an implementation : the body of the module provides an implementation of the sorts and operations of the export interface in terms of those of the import. The export specification is implemented (as in [20]) by the import specification via the constructor functor SEM : Alg(IMP) $\rightarrow$ $A \lg (E X P)$. The transformation induced by the semantics of a module specification can also be viewed as an implementation functor. In general our approach is useful for other notions of implementation, using transformations from Alg(IMP) to $A \lg (E X P)$ other than the semantics of modules, for the constructors in the sense of [20]. The development process using their notion of implementation corresponds to a sequence of direct derivations.

The objective of programming "in the small" is to produce a program (i.e., an element in a language generated by a grammar) which "solves" a problem specified in some formalism. Programming "in the large" is reduced to the same problem, as what is needed is to produce an element of a grammar, keeping track of the "production" process.

ACKNOWLEDGEMENT This research was supported in part by the Consiglio Nazionale delle Ricerche under "Progetto Finalizzato : Sistemi informatici e Calcolo Parallelo" and in part by the Ministero della Pubblica Istruzione

\section{REFERENCES}

[1] E.K.Blum,H.Ehrig,F.Parisi-Presicce,"Algebraic Specification of Modules and their Basic Interconnections", J. Comp. System Sci.34, 2/3 (1987) 239-339

[2] E.K.Blum,F.Parisi-Presicce,"The Semantics of Shared Submodule Specifications", Proc. CAAP85 /TAPSOFT85, Lect. Notes in Comp. Sci. 185, Springer-Verlag 1985, pp.359-373

[3] R.M.Burstall,J.A.Goguen,"Putting Theories together to make Specifications", Proc.5th Int. Conf. on Artificial Intelligence (1977) 1045-1058

[4] R.M.Burstall, J.A.Goguen, "An informal introduction to Specifications using Clear" in "The correctness Problem in Computer Science' (R.S.Boyer, J.S.Moore eds.) Academic Press 1981

[5] B.Krieg-Bruckner, ed., "A Comprehensive Algebraic Approach to System Specification and Development", ESPRIT BRWG 3264, Univ.Bremen, Bericht 6/89 (1989)

[6] H.Ehrig, "Introduction to the Algebraic Theory of Graph Grammars", Lect. Notes in Comp. Sci. 73 (1979) 1-69

[7] H.Ehrig, W.Fey, H.Hansen, M.Lowe, F.ParisiPresicce, "Categories for the Development of Algebraic Module Specifications", Lect. Notes in Comp.Sci. $393(1989)$ 157-184
[8] H.Ehrig, W.Fey, F.Parisi-Presicce, E.K.Blum, "Algebraic Theory of Module Specifications with Constraint", invited, Proc MFCS, Lect. Notes in Comp. Sci. 233(1986) 59-77

[9] H.Ehrig, H.-J.Kreowski, A.Maggiolo-Schettini, B.K.Rosen, J.Winkowski, "Transformation of Structures: An Algebraic Approach", Math. Syst.Theory 14 (1981) 305-334

[10] H.Ehrig, B.Mahr, "Fundamentals of Algebraic Specifications 1: Equations and Initial Semantics", EATCS Monographs on Theoret. Comp. Sci., vol 6, Springer- Verlag 1985

[11] H.Ehrig, B.Mahr, "Fundamentals of Algebraic Specifications 2: Module Specifications and Constraints" EATCS Monographs on Theoret. Comp. Sci., to appear

[12] H.Ehrig, F.Parisi-Presicce, "A Match for Rule Based Modular System Design", in preparation

[13] H.Ehrig, H.Weber, "Algebraic Specification of Modules", in 'Formal Models in Programming' (E.J.Neuhold, G.Chronist, eds.) North-Holland 1985

[14] H,Ehrig, H.Weber, "Programming in the large with Algebraic Module Specifications", in: H.J.Kugler(ed), Information Processing 1986, Amsterdam, NorthHollad 19865/, 675-684

[15] J.A.Goguen, J.Meseguer, "Universal Realization, Persistent Interconnection and Implementation of Abstract Modules", Lect. Notes in Comp. Sci. 140 (1982) 265-281

[16] F.Parisi-Presicce, "Union and Actualization of Module Specifications: Some Compatibility Results", J. Comp. System Sci.35,1 (1987) 72-95

[17] F.Parisi-Presicce, "Partial Composition and Recursion of Module Specifications", Proc TAPSOFT87, Lect. Notes in Comp. Sci. 249 (1987) 217-231

[18] F.Parisi-Presicce, "Product and Iteration of Module Specifications", Proc. CAAP 88 ,Lect. Notes in Comp. Sci. 299 (1988) 149-164

[19] F.Parisi-Presicce, "Modular System Design applying Graph Grammar Techniques", Proc. 16 ICALP, Lect.Notes in Comp. Sci. 372 (1989) 621-636

[20] D.Sannella, A.Tarlecki, "Toward Formal Development of Programs from Algebraic Specifications: Implementation revisited", Lect.Notes in Comp.Sci. 249 (1987) 96-110

[21] D.Sannella, A.Tarlecki, "Toward Formal Development of ML Programs : Foundations and Methodology", U.Edinburgh Tech. Rep. ECS-LFCS-89-71

[22] H.Weber, H.Ehrig, "Specification of Modular Systems", IEEE Trans. on Soft. Eng. SE-12, 7 (1986) 784-798 